\title{
The Performance Of Linear Versus Non-Linear Models In Forecasting Returns On The Johannesburg Stock Exchange
}

\author{
Michael van Gysen, University of Cape Town, South Africa
} Chun-Sung Huang, University of Cape Town, South Africa Ryan Kruger, University of Cape Town, South Africa

\begin{abstract}
In this paper we provide a comprehensive comparison of the predictive accuracy of linear and non-linear models when forecasting financial returns, using a number of macroeconomic variables, on the Johannesburg Stock Exchange. We implement a range of linear specifications, Markov switching ARMA and Dynamic Regression models, and univariate models in which the conditional heteroskedasticity is captured by GARCH or EGARCH innovations. Our results indicate that Markov switching models provide the most significant insample fit. However, results for the stable portion of the out-of-sample period and the recent recovery period are mixed with both EGARCH-based linear models and 2-state Dynamic Regression models outperforming the alternatives. Over the market crisis period we find that the forecast performance of the nonlinear models is worse than that of the linear models, which suggests that the benefit of the nonlinear treatment of conditional volatility diminishes over this period.
\end{abstract}

Keywords: Linear Models; Non-linear Models; Forecasting Returns; Johannesburg Stock Exchange

\section{INTRODUCTION}

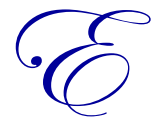

stablishing the predictability of stock market returns has long been the goal of financial practitioners. Advocates of the Efficient Market Hypothesis (EMH) argue that stock prices reflect all publicallyavailable information and therefore do not allow an investor to earn abnormal returns consistently (Fama, 1970). Consequently, the prediction of future stock index returns based on changes in endogenous and exogenous variables that impact on firm performance is redundant, as profit-maximizing agents have already fully incorporated these changes into current prices.

In recent times the veracity of the EMH has been questioned as numerous anomalies have been identified empirically which suggest that predictability in share returns may be related to both endogenous variables such as firm-specific characteristics and exogenous variables such as macroeconomic indicators. These relationships have been approximated using both linear and nonlinear models with varying degrees of success, and the international literature on the perceived forecasting benefits of nonlinear models over linear models has yielded mixed results. Clements and Henry (1988) demonstrate that a superior fit for a nonlinear model in-sample may not yield superior out-of-sample performance. Diebold and Nason (1999) argue that nonlinearities detected in data may result from outliers or structural breaks which cannot necessarily be exploited out-of-sample. They suggest further that nonlinearities related to conditional mean may not be significant enough to yield an improvement in forecasting.

This paper will attempt to distinguish the performance of linear and non-linear models in predicting stock index (ALSI) returns on the Johannesburg Stock Exchange (JSE), using a set of macroeconomic variables. We 
examine a total of fourteen linear and non-linear models for predictive accuracy using a number of out-of-sample forecasting measures.

\section{PRIOR LITERATURE}

Numerous studies have found that asset return series are heteroskedastic, exhibiting time-varying volatility clustering. The autoregressive conditional heteroskedastic (ARCH) model of Engle (1982) and the generalized (GARCH) variant of Bollerslev (1986) both attempt to account for these changes in volatility over time by relating the conditional variance of the series to past variances and observations in the data series. The original GARCH model did not take into account the direction of returns, while the exponential GARCH model (EGARCH) was designed to incorporate the information contained in the signs of the returns. EGARCH takes into account the conditional variance formulation which helps capture the asymmetric response in conditional variance. Alexander (2009) found that EGARCH was the superior model when it came to asymmetric conditional variance as it solves the problem of non-negativity. Su (2010) also found that the EGARCH is superior to the normal GARCH when considering Chinese stock indices over the crisis period.

Gokcan (2000) compared linear GARCH models to non-linear GARCH models on seven different emerging markets and found that linear GARCH models provide better results than non-linear. Another study done by Bonilla and Sepulveda (2011) took 13 emerging markets and used GARCH models to predict stock market indices. They found that the GARCH models failed to capture the time-varying nature of market returns and cautioned their predictive use.

Although GARCH models provide an effective way of modelling non-linear dynamics in stock returns they suffer from one fundamental flaw - they assume that the relationship between excess returns and explanatory factors remains constant no matter what state the economy is in. Periods of 'boom' or market crisis are characterized by substantially higher volatility in returns, which can have a major effect on the parameter estimates of time series models. Schwert (1989) and Hamilton and Lin (1996) found that the volatility of stock returns is higher during recessions compared to expansions. Thus it would seem that different 'regimes' of return and volatility distributions exist in financial time series, and would therefore need to be modelled.

It has been suggested that switching parameters should be used to model the presence of regimes in stock volatility. Without switching parameters, there will be substantial upward bias in the estimates of persistence parameters which occur particularly in high-volatility periods (Gray 1996; Klaassen, 2002). Hamilton (1988) proposed that switching between different regimes should follow a Markov process and subsequently developed a Markov-switching model. Assoe (1998) uses Markov-switching models to examine whether the changes in regime have a significant effect on the returns of shares in 9 emerging markets. Their results show that there is very strong evidence of regime-switching behaviour in emerging markets.

Cai (1994) found that the issue of changing regimes can be resolved by accounting for the rapid, discrete shifts in asymptotic variance by using a Markov process. While Hamilton and Susmel (1994) concluded that their Markov switching specification offered a "better statistical fit to the data and better forecasts", they attributed most of the persistence in stock volatility to the persistence of low, moderate and high volatility regimes which each lasted for several years.

Vast amounts of peer-reviewed literature use macroeconomic variables to explain returns data when using both linear and non-linear models. Guidolin, et al, (2009), Guidolin, et al, (2010), Paye and Timmermann (2006) and McMillan (2003) focused on the use of interest rates, inflation and spot oil prices. All of these studies used nonlinear models such as GARCH and Markov-Switching models to predict returns, along with a focus on developed markets.

Rapach et al (2005) examined the predictability of stock returns using macroeconomic variables and researched which of the commonly proposed variables was most effective in predicting share returns using insample and out-of-sample data in 10 different industrial countries. Their results show that interest rates were the most consistent and reliable macroeconomic predictor of share returns. Pesaran and Timmermann (2000) 
highlighted the negative correlation of inflation and stock returns, which they attributed to inflation's negative correlation with shocks to economic growth and the corresponding positive correlation that share returns have with these shocks. Mpofu, (2009) confirmed this negative relationship between stock returns and inflation within a South African context by examining the inflation stock relationship within 7 developing countries, which have markets similar to that of the JSE.

\section{DATA AND RESEARCH METHOD}

\section{Data}

Price index data for the All Share Index (ALSI) was collected from Bloomberg for the period December 1995 to June 2012. ALSI returns were then calculated as first differences of the logarithmic prices and therefore reflect continuously compounded values.

In addition, data for a range of macroeconomic variables including CPI, PPI, the yield spread between long- and short-term bonds, the rand/dollar exchange rate, the rand/pound exchange rate, the oil price, industrial production, the gold price and the platinum price were collected from Bloomberg over the same period as the ALSI data. We were unable to include unemployment in the list as we were unable to source reliable data extending back to 1995 .

The data was split into an in-sample period encompassing December 1995 to December 2005 and an outof-sample period from January 2006 to June 2012. The out-of-sample period was further sub-divided into three subperiods as detailed in the results section.

\section{Research Method}

The analysis to follow comprises two phases. Firstly, we determine the macroeconomic variables which were most significant in predicting ALSI log returns over our in-sample period using a forward stepwise method. We employ a $p$-value of $5 \%$ for the analysis.

Following this procedure we estimate a range of linear and nonlinear forecasting models and examine the forecasting abilities both in- and out-of-sample. In total we employ 14 models (9 linear and 5 non-linear). As many of these are common in the literature we review them only briefly.

\section{Benchmark Model}

We first consider a simple linear regression that predicts ALSI returns one period ahead using the set of explanatory macroeconomic variables $X_{t-1}$ found to be significant in the stepwise procedure. This can be described by the equation:

$$
r_{t+1}^{A L S I}=c+\left(\beta_{i}\right)^{\prime} X_{t}+\varepsilon_{t+1}
$$

where $c$ is a constant, $\beta_{i}$ is the weighting applied to each macroeconomic variable, and $\varepsilon_{t+l}$ is a martingale difference sequence.

\section{ARMA Models} defined by:

Next we employ autoregressive moving average models, otherwise known as $\operatorname{ARMA}(p, q)$ models, that are

$$
r_{t+1}^{A L S I}=c+\left(\gamma_{i}\right)^{\prime} X_{t}+\left(\theta_{i}\right)^{\prime} \varepsilon_{t}+\varepsilon_{t+1}
$$

where $\gamma_{i}$ are autoregressive coefficients and $\theta_{j}$ are moving average coefficients. For the purpose of this study we employ both an $\operatorname{ARMA}(1,1)$ and an $\operatorname{AR}(1)$ model. Since the mean equation of the return series cannot take into 
account the heteroskedastic effects of a time series often observed in the form of fat tails and volatility clustering, we also apply $\operatorname{GARCH}(1,1)$ innovations to the $\varepsilon_{t}$ terms of the $\operatorname{ARMA}(1,1)$ and $\operatorname{AR}(1)$ models.

\section{GARCH Models}

We also enhance the linear specification to allow for time-varying predictions of asset return volatility to affect the conditional mean forecasts. This leads to the estimation of GARCH-in-mean models described by:

$$
r_{t+1}^{A L S I}=c+\left(\beta_{i}\right)^{\prime} X_{t}+\lambda \hat{\sigma}_{t+1}+\varepsilon_{t+1},
$$

where $\hat{\sigma}_{t+1}$ is the prediction at time $t$ of the volatility of the ALSI log return one period ahead. We also use an alternative specification of the conditional variance in the form of EGARCH. In addition, the residuals are assumed to be either normally or student-t distributed.

\section{Markov Switching Models}

We estimate both Markov-switching dynamic regression models (MS-DR) and Markov-switching auto regression models (MS-ARMA).

The MS-DR specification has the equation:

$$
r_{t+1}=c\left(s_{t}\right)+\boldsymbol{\beta}^{\prime} X_{t}+\varepsilon_{t+1} .
$$

while the MS-ARMA $(1,1)$ model has the equation:

$$
r_{t+1}=c\left(s_{t}\right)+\alpha\left(s_{t}\right) r_{t}+\lambda\left(s_{t}\right) \mu_{t}+\boldsymbol{\beta}^{\prime} X_{t}+\varepsilon_{t+1},
$$

where $\varepsilon_{\mathrm{t}} \sim \mathrm{N}\left(0, \sigma^{2}\right)$ for both equations. As is evident in the MS-DR model, only constant $c$ is dependent on the unobservable state variable $s_{t}$. Whereas in the MS-ARMA model, the constant $c$, the autoregressive component $\alpha$, and the moving average component $\lambda$ all depend on the unobservable state variable $s_{t}$.

The MS-DR model is a simple regression equation with no autoregressive or moving average variables. For both models we allow for switching in the error variance (heteroskedasticity) which leads to the expansions MSHDR and MSH-ARMA.

The likelihood of both models is evaluated using the filtering procedure of Hamilton (1989), followed by the smoothing algorithm of Kim (1994). The log-likelihood, which is a function of the parameters in equations (4) and (5) as well as the transition probabilities $p_{i \mid j}$, are then maximised subject to the constraint that the probabilities lie between 0 and 1 and sum to unity. Most literature suggests using the EM algorithm of Dempster, Laird and Rubin (1977) to maximise the log-likelihood, however we prefer the more robust feasible sequential quadratic programming (SQPF) technique of Lawrence and Tits (2001). The covariance matrix is estimated using second derivatives.

\section{In-Sample Measures of Performance}

We employ three information criteria as a guide to selecting the model with the best in sample fit - the Akaike Information Criterion (AIC) of Akaike (1974), the Bayesian Information Criterion (BIC) of Schwarz (1978) and the Hannan-Quinn Criterion (HQ) of Hannan and Quinn (1979).

\section{Out-of-Sample Measures of Performance}

Models are constructed using a recursive procedure with an expanding window. The recursive procedure estimates the model over the in-sample period and then uses it to forecast the first observation in the out-of-sample period. This observation is then added to the estimation sample and the model is re-estimated and used to forecast the second observation in the out-of-sample period. This process is repeated until the final observation in the out-ofsample period has been forecast. 
We define the forecast error at time $t$, from model $i$, at horizon $h$ as:

$$
e_{t, t+h}^{i}=r_{t}^{A L S I}-\hat{r}_{t, t+h}^{i}
$$

where $\hat{r}_{t, t+h}^{i}$ is the forecast from any of the fourteen models described above. For each model we compute five different measures of predictive accuracy: Root Mean Square Forecast Error (RMSFE), Forecast Error Bias (FEB), Forecast Error Variance (FEV), Mean Absolute Forecast Error (MAFE) and Mean Percentage Forecast Error (MPFE).

\section{EMPIRICAL RESULTS}

\section{Stepwise Procedure}

We ran the stepwise regression using lagged variables of 1,2 and 3 months for all 9 macroeconomic variables listed above, resulting in a list of 27 explanatory variables. The dependent variable was the ALSI log return for monthly data from 1995:12 - 2005:12, for a total of 122 observations.

The stepwise regression indicated that the most significant variables for the period were the Oil Price and the Yield Spread, both lagged by 1 month. Thus the series used in all models described prior are the ALSI log returns $\left(r_{t}^{A L S I}\right)$ the oil price $\left(\right.$ oil $\left._{t-1}\right)$ and the lagged yield spread variable $\left(\operatorname{spread}_{t-1}\right)$.

\section{Model Evaluation}

\section{In-Sample Results}

Table 1 presents statistics for the information criteria with regards to the in-sample fit of all fourteen models. The lower the statistic the better the model fits the data; the two smallest values for each criterion have been boldfaced. It is quite clear that the addition of regime switching models has resulted in a greater in-sample fit over the simple linear specifications. In particular, the 2-state MS-DR model features within the top two models for each of the criterion evaluated.

Table 1: Goodness-Of-Fit Tests For The Period 1995:12 - 2005:12

\begin{tabular}{|l|c|c|c|}
\hline \multicolumn{1}{|c|}{ Model } & AIC & Schwarz Criterion & Hannan-Quinn Criterion \\
\hline Simple Regression & -2.64099 & $\mathbf{- 2 . 5 7 1 6 7}$ & -2.61284 \\
\hline AR(1) & -2.62575 & -2.53283 & -2.58801 \\
\hline AR(1) with GARCH(1,1) & -2.67899 & -2.51638 & -2.61295 \\
\hline ARMA(1,1) & -2.65938 & -2.54323 & -2.61221 \\
\hline ARMA(1,1) with GARCH(1,1) & -2.68868 & -2.50285 & -2.61322 \\
\hline GARCH(1,1)-in-mean & -2.69085 & -2.52911 & -2.62516 \\
\hline EGARCH(1,1)-in-mean & -2.74185 & -2.55701 & -2.66678 \\
\hline GARCH(1,1)-in-mean (tdist) & -2.71354 & -2.52870 & -2.63847 \\
\hline EGARCH(1,1)-in-mean (tdist) & -2.69191 & -2.48396 & -2.60745 \\
\hline MS-ARMA(1,1) (2 States) & -2.58710 & -2.33158 & -2.48333 \\
\hline MSH-ARMA(1,1) (2 States) & -2.71652 & -2.46100 & -2.61275 \\
\hline MS-DR (2 States) & $\mathbf{- 2 . 7 5 9 4 3}$ & $\mathbf{- 2 . 6 2 0 8 0}$ & $\mathbf{- 2 . 7 0 3 1 3}$ \\
\hline MS-DR (3 States) & -2.74338 & -2.53543 & -2.65892 \\
\hline MS-ARMA(1,1) - 3 States & $\mathbf{- 2 . 8 5 8 6 4}$ & -2.51020 & $\mathbf{- 2 . 7 1 7 1 4}$ \\
\hline
\end{tabular}

\section{Out-of-Sample Results}

Table 2 provides a summary of predictive accuracy for each model over the full out-of-sample period according to the five performance measures described in our research method. The two best performance figures for each statistic have been boldfaced. Results over the period are mixed with both the EGARCH-in-mean (Gaussian) and 2-state MS-DR models demonstrating lower forecasting errors and a superior ability to predict the direction of returns relative to the alternative specifications. 
Table 2: One-Period Ahead Forecast Measures For The Period 2006:01 To 2012:06

\begin{tabular}{|l|c|c|c|c|c|c|}
\hline \multicolumn{1}{|c|}{ Model } & RMSFE & FEB & FEV & MAFE & MPFE & Success Ratio \\
\hline Simple Regression & 0.05284 & -0.00586 & 0.00276 & 0.04141 & 1.60567 & 0.5128 \\
\hline AR(1) & 0.05340 & -0.00548 & 0.00282 & 0.04186 & 1.71977 & 0.5385 \\
\hline AR(1) with GARCH(1,1) & 0.05270 & -0.00541 & 0.00275 & 0.04156 & 1.81725 & 0.4872 \\
\hline ARMA(1,1) & 0.05525 & $\mathbf{- 0 . 0 0 2 7 2}$ & 0.00304 & 0.04364 & 1.76564 & 0.5000 \\
\hline ARMA(1,1) with GARCH(1,1) & 0.05254 & -0.00380 & 0.00275 & 0.04155 & 1.79597 & 0.5128 \\
\hline GARCH(1,1)-in-mean & 0.05236 & -0.00449 & $\mathbf{0 . 0 0 2 7 2}$ & 0.04158 & 1.69310 & 0.4744 \\
\hline EGARCH(1,1)-in-mean & $\mathbf{0 . 0 5 2 1 8}$ & $\mathbf{0 . 0 0 1 0 8}$ & $\mathbf{0 . 0 0 2 7 2}$ & 0.04161 & 1.44121 & 0.4872 \\
\hline GARCH(1,1)-in-mean (tdist) & 0.05346 & -0.00498 & 0.00283 & 0.04200 & 1.68726 & 0.4744 \\
\hline EGARCH(1,1)-in-mean (tdist) & 0.05467 & -0.00654 & 0.00295 & $\mathbf{0 . 0 4 0 1 2}$ & 1.63874 & $\mathbf{0 . 5 7 6 9}$ \\
\hline MS-ARMA(1,1) (2 States) & 0.06300 & -0.00809 & 0.00390 & 0.04879 & $\mathbf{1 . 3 1 7 5 9}$ & 0.5000 \\
\hline MSH-ARMA(1,1) (2 States) & 0.05996 & -0.00479 & 0.00357 & 0.04839 & 1.60430 & $\mathbf{0 . 5 6 4 1}$ \\
\hline MS-DR (2 States) & $\mathbf{0 . 0 5 2 1 5}$ & -0.00499 & $\mathbf{0 . 0 0 2 7 0}$ & $\mathbf{0 . 0 3 9 8 4}$ & 1.47495 & 0.5385 \\
\hline MS-DR (3 States) & 0.05260 & -0.00470 & 0.00274 & 0.04064 & $\mathbf{1 . 4 1 2 9 7}$ & 0.5128 \\
\hline MS-ARMA(1,1) (3 States) & 0.07587 & -0.00591 & 0.00572 & 0.05840 & 1.71558 & 0.4744 \\
\hline
\end{tabular}

The continued superior performance of the 2-state MS-DR model is surprising given that prior research has indicated that non-linear models tend to over-fit the data in-sample and hence do not perform consistently out-ofsample. These results also provide an interesting contrast to those of the MS-ARMA based models, which are outperformed by the linear model specifications. This could be as a result of the sample size not being large enough for these models to generate accurate estimations for each parameter and they may therefore be describing the random error or noise, and not the underlying relationship. The problem seems to be specific to the MS-ARMA models that estimate far more parameters than the MS-DR models, which only have switching in the constant.

As can be seen in Figure 1, the period (2006:01 to 2012:06) over which we sampled the forecast errors includes the extremely volatile period of the recent financial crisis. It may be true that certain models perform well during relatively stable periods, while others perform during unstable periods.

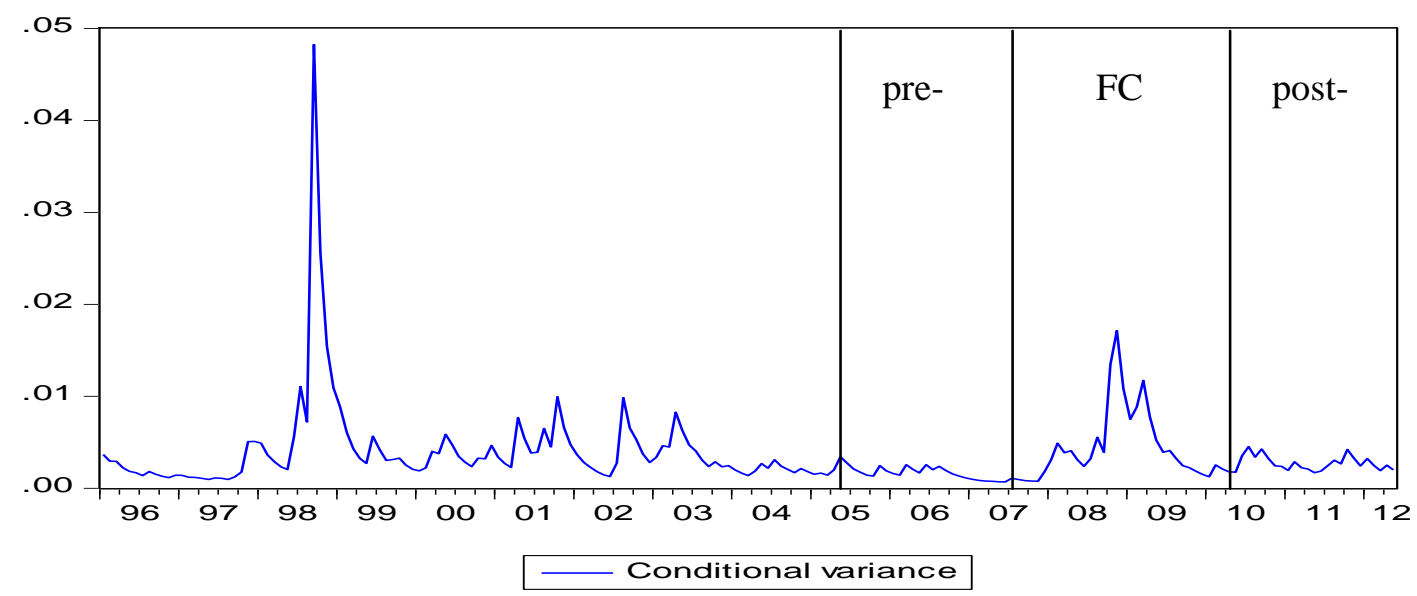

Figure 1: Graph Of The Conditional Variance Of ALSI Log Returns For The Period 1996:01 To 2012:06

This prompts us to replicate the analysis for periods pre- (2006:01 - 2007:12), during (2008:01 - 2009:02) and post- (2009:03 - 2012:06) the financial crisis (FC). These results are displayed in Table 3.

The sub-period analysis presents an interesting series of results. Over the stable 24-month period prior to the crisis, we find that the $\operatorname{EGARCH}(1,1)$-in-mean model (t-distribution) outperforms across all 5 forecasting criterion and yields a success ratio of 79.17\%. The MS-DR models follow next with the 2- and 3-state models yielding very similar results but the 3 -state model marginally ahead with regards to minimizing forecasting errors and the 2-state providing a higher success rate. 
Over the crisis period, we find that the linear models demonstrate better forecasting performance with models including an $\operatorname{ARMA}(1,1)$ specification being preferred. This is more consistent with earlier findings regarding the weak out-of-sample performance of nonlinear models (see for example Clements and Henry, 1988 and Dieboldt and Nasan, 1999). Furthermore, the Student $t$-distribution is outperformed by the normal distribution for the various GARCH models. We extended the GARCH model to the $t$-distribution in order to take into account the presence of leptokurtosis and fat tails in the returns. Thus it would seem that the added volatility of this period has resulted in greater deviations from the mean, with less outlying events, resulting in a distribution of returns that is less leptokurtic and more normally distributed.

During the recovery period of the sample, we again find that the $\operatorname{EGARCH}(1,1)$-in-mean model (tdistribution) and 2-state MS-DR model outperform the alternatives tested. EGARCH takes into account the leverage effect, thus its success would suggest the importance of using the vital information contained within the signs of the returns. One could hypothesise that the pessimism felt as a result of the financial crisis, would lead investors to panic and trade more positions when asset prices decreased, compared to when asset prices increased.

\section{CONCLUSION}

In this paper we provide a comprehensive comparison of the predictive accuracy of linear and non-linear models using a number of macroeconomic variables. We consider a variety of linear models including ARMA variations, GARCH, and EGARCH models which allow for ARCH-in-mean effects. Examining MS-ARMA and MS-DR models extends the paper to include non-linear specifications. Prior literature highlights the superiority of non-linear models in forecasting returns in developed markets, with there being little research into emerging markets, specifically the JSE.

Our results indicate that 3-state MS models provide the most significant in-sample fit. This is followed by the 2-state MS-DR. However when examining out-of-sample forecasts, the results vary depending on the state of the economy. During the 2-year stable period immediately preceding the market crisis and the market recovery period evidenced from 2009, we find that the EGARCH(1,1)-in-mean (t-distributions) and MS-DR models are the most consistent. In contrast, the linear model specifications are most appropriate during the market crisis, particularly those incorporating a $\operatorname{ARMA}(1,1)$ term. 
Table 3: One-Period Ahead Forecast Measures For Sub-Periods Within The Out-Of-Sample Period

\begin{tabular}{|c|c|c|c|c|c|c|c|c|c|c|c|c|c|c|c|c|c|c|}
\hline \multirow[b]{2}{*}{ Model } & \multicolumn{6}{|c|}{ 2006:01 to 2007:12 } & \multicolumn{6}{|c|}{ 2008:01 to 2009:12 } & \multicolumn{6}{|c|}{ 2010:01 to 2012:12 } \\
\hline & RMSFE & FEB & FEV & MAFE & MPFE & $\begin{array}{c}\text { Success } \\
\text { Ratio }\end{array}$ & RMSFE & FEB & FEV & MAFE & MPFE & $\begin{array}{c}\text { Success } \\
\text { Ratio }\end{array}$ & RMSFE & FEB & FEV & MAFE & MPFE & \begin{tabular}{|c} 
Success \\
Ratio
\end{tabular} \\
\hline Simple Regression & 0.038 & 0.019 & 0.001 & 0.032 & 1.251 & 0.500 & 0.072 & -0.013 & 0.005 & 0.055 & 1.795 & 0.583 & 0.044 & -0.020 & 0.002 & 0.038 & 1.738 & 0.467 \\
\hline AR(1) & 0.039 & 0.022 & 0.001 & 0.033 & 1.319 & 0.542 & 0.073 & -0.013 & 0.005 & 0.055 & 2.119 & 0.625 & 0.044 & -0.021 & 0.002 & 0.038 & 1.721 & 0.467 \\
\hline AR(1) with GARCH $(1,1)$ & 0.040 & 0.018 & 0.001 & 0.035 & 1.257 & 0.458 & 0.072 & -0.017 & 0.005 & 0.055 & 2.562 & 0.542 & 0.042 & -0.015 & 0.002 & 0.036 & 1.670 & 0.467 \\
\hline $\operatorname{ARMA}(1,1)$ & 0.046 & 0.030 & 0.001 & 0.038 & 1.500 & 0.417 & 0.074 & -0.013 & 0.005 & 0.056 & 2.076 & 0.625 & 0.044 & -0.021 & 0.002 & 0.038 & 1.730 & 0.467 \\
\hline $\begin{array}{l}\operatorname{ARMA}(1,1) \text { with } \\
\operatorname{GARCH}(1,1)\end{array}$ & 0.042 & 0.021 & 0.001 & 0.037 & 1.300 & 0.500 & 0.071 & -0.016 & 0.005 & 0.053 & 2.435 & 0.583 & 0.042 & -0.014 & 0.002 & 0.036 & 1.682 & 0.467 \\
\hline $\operatorname{GARCH}(1,1)$-in-mean & 0.040 & 0.018 & 0.001 & 0.035 & 1.167 & 0.417 & 0.071 & -0.012 & 0.005 & 0.054 & 2.195 & 0.542 & 0.043 & -0.016 & 0.002 & 0.037 & 1.713 & 0.467 \\
\hline EGARCH(1,1)-in-mean & 0.042 & 0.021 & 0.001 & 0.036 & 1.167 & 0.500 & 0.070 & -0.006 & 0.005 & 0.055 & 1.577 & 0.500 & 0.042 & -0.009 & 0.002 & 0.036 & 1.552 & 0.467 \\
\hline $\operatorname{GARCH}(1,1)$-in-mean (tdist) & 0.039 & 0.019 & 0.001 & 0.035 & 1.234 & 0.375 & 0.074 & -0.016 & 0.005 & 0.057 & 2.210 & 0.583 & 0.042 & -0.015 & 0.002 & 0.036 & 1.632 & 0.467 \\
\hline 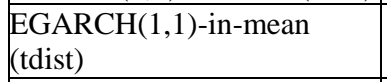 & 0.032 & 0.007 & 0.001 & 0.025 & 0.570 & 0.792 & 0.081 & -0.017 & 0.006 & 0.061 & 2.899 & 0.500 & 0.041 & -0.009 & 0.002 & 0.035 & 1.485 & 0.467 \\
\hline MS-ARMA(1,1) (2 States) & 0.039 & 0.008 & 0.001 & 0.033 & 1.189 & 0.542 & 0.089 & -0.003 & 0.008 & 0.070 & -0.200 & 0.500 & 0.052 & -0.025 & 0.002 & 0.045 & 2.634 & 0.467 \\
\hline MSH-ARMA(1,1) (2 States) & 0.040 & 0.015 & 0.001 & 0.032 & 1.182 & 0.583 & 0.082 & -0.007 & 0.007 & 0.067 & 1.052 & 0.625 & 0.052 & -0.019 & 0.002 & 0.046 & 2.384 & 0.500 \\
\hline MS-DR (2 States) & 0.036 & 0.017 & 0.001 & 0.030 & 0.964 & 0.583 & 0.074 & -0.015 & 0.005 & 0.056 & 2.058 & 0.583 & 0.041 & -0.015 & 0.001 & 0.034 & 1.417 & 0.467 \\
\hline MS-DR (3 States) & 0.035 & 0.014 & 0.001 & $\mathbf{0 . 0 3 0}$ & 0.927 & 0.542 & 0.074 & -0.015 & 0.005 & 0.058 & 1.801 & 0.542 & 0.043 & -0.012 & 0.002 & 0.036 & 1.491 & 0.467 \\
\hline MS-ARMA $(1,1)$ - 3 States & 0.045 & 0.011 & 0.002 & 0.036 & 1.354 & 0.458 & 0.099 & 0.000 & 0.010 & 0.080 & 1.701 & 0.667 & 0.074 & -0.024 & 0.005 & 0.059 & 2.017 & 0.333 \\
\hline
\end{tabular}




\section{AUTHOR INFORMATION}

Michael van Gysen graduated from the University of Cape Town with a Bachelor of Business Science, specialising in the field of Finance. He is currently furthering his studies in the field of Mathematics and Statistics at the same institution. Email: michaelvangysen@gmail.com

Chun-Sung Huang is a lecturer in Finance and an associate of the African Collaboration for Quantitative Finance and Risk Research (ACQuFRR) at the University of Cape Town, South Africa. His research interests lie in volatility modelling and forecasting, Value-at-Risk (VaR) models and derivatives pricing in incomplete markets. Email: Chun-Sung.Huang@uct.ac.za (Corresponding Author)

Ryan Kruger is a Senior Lecturer in Finance and is an associate and founding member of the African Collaboration for Quantitative Finance and Risk Research (ACQuFRR) at the University of Cape Town, South Africa. His research interests lie in the predictive modelling of asset returns and the application of machine learning in asset pricing. Email: ryan.kruger@uct.ac.za

\section{REFERENCES}

1. Akaike, H. (1974). A new look at the statistical model identification. IEEE Transactions on Automatic Control, 19(6), 716-723.

2. Alexander, C. (2009). Practical Financial Econometrics. s.1.:John Wiley \& Sons, Ltd.

3. Assoe, K. (1998). Regime-Switching in Emerging Stock Market Returns. Multinational Finance Journal. 2 (2), 101-133

4. Bollerslev, T. (1986). Generalized Autorregressive Conditional Heteroskedasticity. Journal of Econometrics, 31, 307-327.

5. Bonilla, C \& Sepúlveda, J. (2011). Stock returns in emerging markets and the use of GARCH models. Applied Economics Letters, 18:14, 1321-1325.

6. Cai, J. (1994). A Markov Model of Switching-Regime ARCH. Journal of Business and Economics Statistics, 12, 309-316.

7. Clements, M. P. and Hendry, D. F. (1998). Intercept corrections and structural change. Journal of Applied Econometrics, 11, 475-494.

8. Dempster, A., Laird, N. \& Rubin, D. (1977). Maximum likelihood from incomplete. Journal of the Royal Statistical Society, 39(Series B), 1-22.

9. Diebold, F. X and Nason, J. A. (1990). Nonparametric Exchange Rate Prediction?, Journal of International Economics, 28 (3-4), 315-332.

10. Engle, R. (1982). Autoregressive Conditional Heteroskedasticity with Estimates of United Kingdom Inflation. Econometrica, 50, 987-1008.

11. Fama, E. (1970). Efficient Capital Markets: A Review of Theory and Empirical Work. The Journal of Finance, 25(2), 383-417.

12. Gokcan, S. (2000). Forecasting Volatility of Emerging Stock Markets: Linear versus Non-linear GARCH Models. Journal of Forecasting, 19, 499-505.

13. Gray, S.F. (1996). Modeling the conditional distribution of interest-rates as a regime-switching process. Journal of Financial Economics, 3, 59-71.

14. Guidolin, M., Hyde, S., McMillan, D. \& Ono, S. (2009). Non-linear predictability in stock and bond returns: When and where is it exploitable? International journal of forecasting, 25(2), 373-399

15. Guidolin, M., Hyde, S., McMillan, D. \& Ono, S. (2010). Does the Macro economy Predict U.K. Asset Returns in a Nonlinear Fashion? Comprehensive Out-of-sample Evidence. Federal Reserve Bank of St. Louis: Working Paper Series. 2010-039A

16. Hamilton, J.D. \& Lin, G. (1996). Stock market volatility and the business cycle. Journal of Applied Econometrics, 11, 573-593.

17. Hamilton, J. D. (1988). Rational-Expectations Econometric Analysis of Changes in Regime. An Investigation of the Term Structure of Interest Rates. Journal of Economic Dynamics and Control, 12, 385-423.

18. Hamilton, J. D. (1989). A New Approach to the Economic Analysis of Nonstationary Time Series and the Business. Econometrica, 57(2), 357-384. 
19. Hamilton, J.D. \& Susmel, R. (1994). Autoregressive conditional heteroskedasticity and changes in regime. Journal of Econometrics, 64, 307-333.

20. Hannan, E. \& Quinn, B. (1979). The Determination of the Order of an Autoregression. Journal of the Royal Statistical Society, 41(2), 190-195.

21. Kim, C.-J., (1994). Dynamic linear models with Markov-switching. Jornal of Econometrics, 60(1/2), 1-22.

22. Klaassen, F. (2002). Improving GARCH volatility forecasts with regime-switching GARCH. Empirical Economics, 27, 363-394.

23. Lawrence, C. \& Tits, A. (2001). A computationally efficient feasible sequential quadratic. SIAM Journal on Optimisation, 11(4), 1092-1118.

24. McMillan, D.G. (2003). Non-linear Predictability of UK Stock Market Returns. Oxford bulletin of economics \& statistics. 65(5), 557-573.

25. Mpofu, B. (2009). The relationship between stock market returns and inflation: new evidence from sub-saharan Africa. University of St. Andrews

26. Paye, B.S. \& Timmermann, A. (2006). Instability of return prediction models. Journal of empirical finance. 13(3), 274-315.

27. Pesaran, M.H. \& Timmermann, A. (2000). A recursive modelling approach to predicting UK stock returns', The Economic Journal, 110, 159-191.

28. Rapach, D,E. \& Wohar, M.E. \& Rangvid, J. (2005). Macro variables and international stock return predictability. International Journal of Forecasting 21, 137-166

29. Schwert, G.W. (1989). Whydoes stock market volatilitychange over time?. Journal of Finance, 44, 1115-1153.

30. Schwarz, G. (1978). Estimating the dimension of a model. Annals of Statistics, 6(2), 461 - 464.

31. Su, C. (2010). Application of EGARCH Model to Estimate Financial Volatility of Daily Returns: The empirical case of China. University of Gothenburg. Project No. 2010:142. 